\title{
Visible Imagination: projected play
}

\author{
David J. Chatting, Jon S. Sutton \\ Media Interfaces Group \\ BT Innovate \\ Adastral Park, Martlesham Heath \\ Suffolk, UK \\ +441473648639 \\ david.chatting@gmail.com, jon.sutton@gmail.com
}

\begin{abstract}
This paper presents a system for the exploration of projected interfaces. We describe our Visible Imagination (VI) platform combining a camera, projector and torch (flashlight) allowing interaction with the torchlight and shape capture. We describe six application sketches that demonstrate different interaction styles using the platform, reflecting on their success and how they may be applied to play.
\end{abstract}

\section{Categories and Subject Descriptors}

H.5.2 [Information Interfaces and Presentation]: User Interfaces - evaluation/methodology, user-centred design, prototyping.

\section{General Terms}

Design, Human Factors.

\section{Keywords}

Projected interfaces, Torches, Flashlights, Children's Toys, Play

\section{INTRODUCTION}

As the cost and size of computing for computer-vision and image projection continues to fall, we believe this technology will soon be adopted in children's toys. There is the potential to allow creative, imaginative play beyond the monitor-keyboardmouse paradigm with more active, collaborative and tangible play.

The projector is now a ubiquitous part of any professional presentation suite and is gradually being seen in more domestic environments for film and television viewing. In general these systems project onto a specially prepared surface, usually matte white. However, with the brightness of modern projectors as it is, many surfaces and objects can show an image, allowing an augmentation of the real with the virtual.

The Visible Imagination (VI) prototype is a platform for exploring projected interfaces for children's play in the home.

(C) The Author 2009 .

Published by the British Computer Society
It comprises a collocated camera and projector with a PC to process the image from the camera and drive the projected graphics. A torch (flashlight), equipped with Bluetooth radio, enables a beam of light to be shone into the scene and buttons to be pressed to initiate actions. Two styles of user interaction are supported by VI, using the beam of a torch and using a hand or other prop to create shapes to capture.

We are interested in dark and semi-light environments where imagination and storytelling thrives [12].

Our aim is to find new ways to build toys that are fun, and encourage open-ended imaginative play. Our work has not been concerned specifically with educational outcomes, although this is clearly relevant.

In this paper we describe six application sketches that demonstrate different interaction styles using the VI platform. The next section describes related work; the platform design, application sketches and a discussion of these interactions conclude.

\section{RELATED WORK}

Toy theatres, shadow puppets and magic lanterns have been used in storytelling for hundreds of years, delighting generations of children. Recently this tradition has been reinterpreted in works such as Takashi's Seasons [4] and Worthington's Shadow Monsters [16].

Takashi's Seasons [4] is a shadow show in which the silhouettes of real puppets are augmented with computer-generated imagery. For instance, the trees drop their leaves and the flowers bloom. The puppeteers must trigger these animations and move the puppet in perfect synchrony for the effect to realised.

Worthington's Shadow Monsters [16] augments traditional hand shadow puppets with virtual features, such as adding a tongue and scales to a hand forming a monster's head. In realtime the system processes video images to extract silhouettes of hands. By applying rules about the nature of these silhouette's outline (concave, convex, enclosed, etc.) additions are then made and the image projected. Unlike [4] the whole image is generated, the hand's real shadow is not visible. However, where the camera and projector are well configured the illusion of direct manipulation remains.

While Shadow Monsters [16] demonstrates captured silhouettes, I/O Brush [10] extends this for colours and textures.

I/O Brush [10] uses a physical brush and touch-screen for interaction, allowing children to capture colours and textures from their environment, to paint with them. The brush contains 
a light and camera, to illuminate and capture the surface. When the brush is applied to a touch-sensitive display it appears to paint with the captured texture. This encourages the child to explore their environment - to look at the world in a new way.

Selective illumination by a laser or torch (flashlight) for input has been previously explored by a number of people, for instance $[1,2,7,8,14]$.

Laser pointers are an attractive option for the apparent precision they allow and when pointing over a large distance, as the beam stays focused [8]. This is exemplified by Laser Tag 2.0 [14]. However, there are some legitimate safety concerns with lasers, especially for a child's use.

While a torch in comparison to a laser is relatively dim; over the short distances expected in the home, the bright light gives enough illumination to be reliably tracked by a camera and computer-vision techniques. Less powerful torches may be successfully used in semi-dark environments.

Ghali et al [1] used torches to trigger audio clips associated with real objects in the space, such that when they were illuminated the sound would be triggered.

Story Tent [2] investigated the combination of projected imagery and torch interaction for young children. Children inside the tent see the rendering of a 3D world that they can manipulate with their torches. This interaction allowed virtual objects, such as balloons to be moved around the world. Tracking multiple beams allowed collaborative play.

The torch is a simple and safe tangible device that has a number of interesting properties: a pool of light where the area changes with distance and an elliptical shape changing with angle, the brightest area being in the centre gradually diminishing at the edges. See Ghali et al [1] for a comprehensive discussion of the properties of torches for interaction.

In O'Shea's "Out of Bounds" installation [7] an invisible infrared light as used to interact with a projected image. This is attractive as it allows the appearance of the light to be drawn virtually on the wall in whichever way is appropriate. However, the projected image creates a cone of light from the point of the projector, rather than from the torch itself. We also prefer visible light as it allows any torch or source of light to be appropriated by the child in play.

Many of these systems assume a flat fixed projection surface and as a result are troubled by occlusions. Multiple projector solutions to this have been developed that reduce these effects, for example GVU-PROCAMS [11]. However, we prefer to think of the projection surface as irregular and dynamic. The "It's fire, you can touch it" installation [3] illustrates how objects (in this case hands) can be moved within the volume of the projection and be augmented with graphics in real-time.

Westerdiep's de Pong game [13] also demonstrates how projection allows real objects in a $3 \mathrm{D}$ scene to be augmented. A large game of pong is shown on the side of a building, from which features such as windows and doors are extracted, so that the puck appears to interact with the architecture.

Wilson's PlayAnywhere [15] uses a short-throw projector and camera combination for tabletop displays. Interaction is achieved with hand gestures, including touch, and with real objects, for example a sheet of paper or mobile telephone.

The Visible Imagination platform seeks to build on and extend the related work identified here.

\section{VISIBLE IMAGINATION PLATFORM}

This section describes the hardware and software of the Visible Imagination (VI) platform developed, on which our experimental sketches were built.

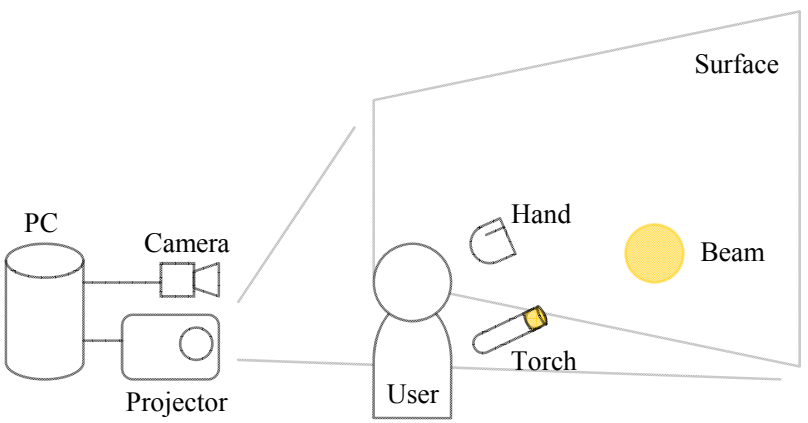

Figure 1 Visible Imagination physical configuration

\subsection{Hardware}

The hardware configuration we choose for VI was that of the camera, projector and torch. We envisage the system integrated into a wireless, battery powered unit that can be placed anywhere the child wants to play. As such the camera and projector are collocated, as shown in Figure 1. Alternative designs avoid occlusion of view by placing the camera in a higher position [7] or with the projector and/or camera on the other side of a semitransparent display screen [16].

The camera used is an Apple iSight Firewire camera capturing frames at a resolution of 640 by 480 pixels. Located with the projector, the camera's view should include all of the projected area and will typically see a boundary around this.

The projector used was a Sony VPL EX4, set to a resolution of 800 by 600 , with a maximum output of 2100 lumens. For best results the brightness was decreased such that when showing black the wall was not visibly illuminated; graphics on a black background are more seamlessly augmented into the scene without a visible projection boundary. The image is projected directly onto surfaces within the environment, without a special screen. During development the camera and projector were typically set back 1.5 metres from the wall, showing an image of 1 metre (corner to corner).

The torch contains a single ultra-bright white LED and a Bluetooth radio module to communicate button presses to the PC. The topmost button turns the light on and off, the square button selects an option and the circle button returns to the previous menu. See Figure 2.

The $\mathrm{PC}$ is a $3.2 \mathrm{GHz}$ machine running Windows $\mathrm{XP}$.

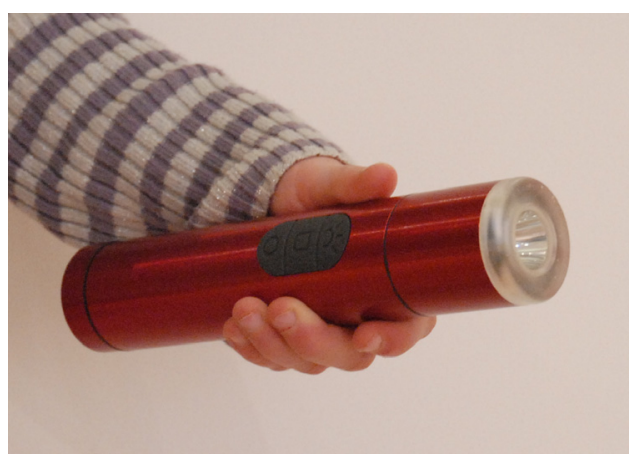

Figure 2 The Visible Imagination torch 


\subsection{Software}

The software architecture shown in Figure 3 consists of two major layers: the application logic written in Flash ActionScript and the image processing implemented in $\mathrm{C}++$ using the OpenCV libraries. The use of $\mathrm{C}++$ gives the performance required to track the torch in real-time; at up to 30 frames per second on the current hardware. The two layers communicate via an XML socket and uses the file system to exchange data files.

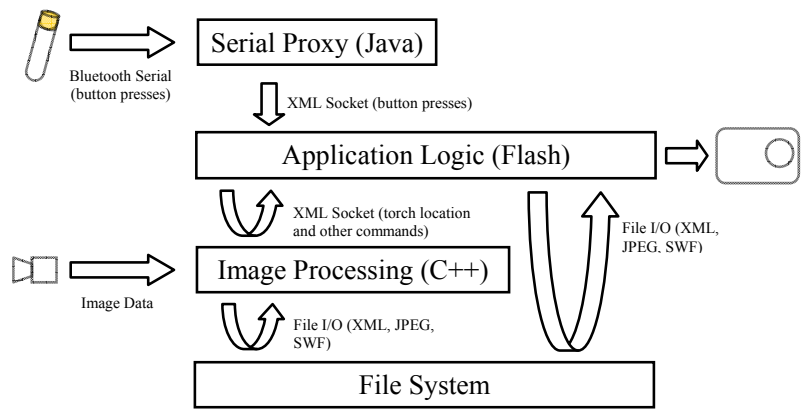

Figure 3 Software architecture

The Image Processing layer provides operations that report the location of the torch beam. The beam must be the brightest spot in camera's view, as such the projected image should not be brighter. Currently only one beam can be tracked at a time.

Calibration allows an observation made in the camera's coordinate system to be mapped into the Flash application space, such that a graphic may be placed to appear coincidently with the beam. In addition, operations allow vector shapes to be captured from within specified areas of the foreground or background. These are encoded as Flash movies and exchanged via the file system. Background modelling to allow the extraction of foreground objects is handled automatically, accumulating a running mean to adjust for changing lighting conditions. The Application Logic layer can force the background to be reset in the case that there is a dramatic change in the scene; it can also change the parameters of all image processing operations dynamically.

Button presses from the Bluetooth torch are reported to the Flash application via a serial-proxy socket server.

\section{APPLICATION SKETCHES}

This section describes the six application sketches developed on the Visible Imagination platform, each demonstrating a different interaction style.

\subsection{Menu}

The Menu allows the selection of the five other sketches; see Figure 4. As the torch beam is shone at each item they are enlarged to show the selection and a different note is played. By pressing the square button on the torch the selected sketch is started.

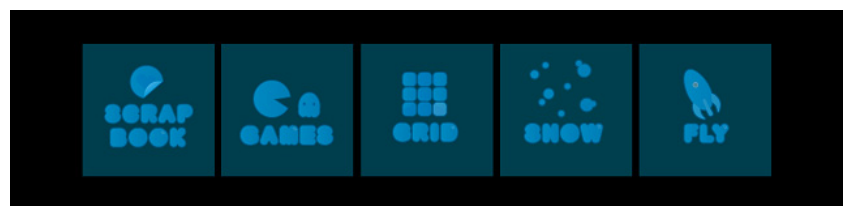

Figure 4 Menu

\subsection{Scrapbook}

The scrapbook demonstrates the simplest interaction with the torch; by shining the beam at the on-screen graphics and holding the square button on the torch they can be dragged and dropped around the space. See Figure 5.

A hit is established when the centre of the tracked torch beam collides with any of the on-screen objects.

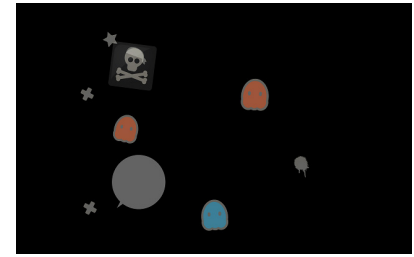

Scrapbook

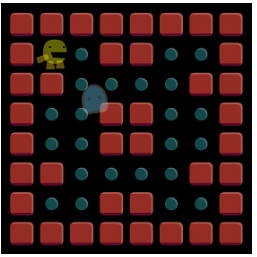

Maze Game
Figure 5 Screenshots from the Scrapbook and Maze Game

\subsection{Maze Game}

The game is similar in concept to Pacman (see Figure 5) involving eating the dots without being caught by the ghost. As the character moves incrementally and relative to its last position, the torch position must be translated into the commands up, down, left or right. We calculate the torch's polar coordinates relative to the centre of the maze and quantise this angle to generate the movement commands. Alternatively this could be calculated relative to the character's position, but in that case the effect of shining the torch at the same area of the screen would be inconsistent over time. In this way the torch is shone around the circumference of the maze to move the character.

\subsection{Snow}

Snow shows an interaction between real objects and projected graphics, as exemplified previously by Westerdiep's de Pong game [13].

By capturing a background image, the edges of objects within the projection area can be extracted and mapped into the application space (see Figure 6). A vector-based description of the scene is created using OpenCV's Canny edge-detection (cvCanny) and contour extraction (cvFindContours) functions. The result is encoded in the Flash movie format (swf) using the Winming library. If projected, the lines in this movie would lay on their real-world counterparts.

This enables an animation, in this case falling snow, to interact with real objects in the scene.

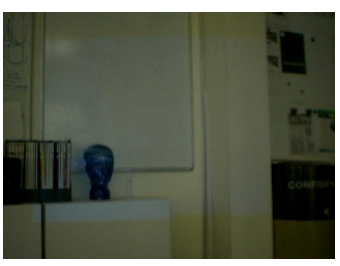

Background

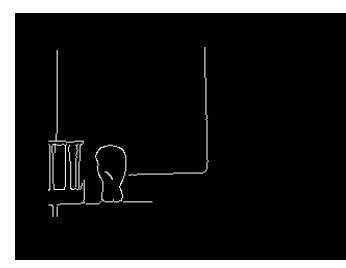

On-screen edges in camera space

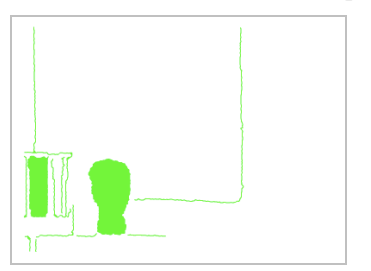

On-screen edges in application space (swf)

Figure 6 Extraction of background edges 


\subsection{Fly}

Fly takes real objects in the scene and copies them into the virtual world. Inspired by Ryokai's I/O Brush [10], we wanted to encourage children to make or find interesting shapes and textures in their environment and to 'bring wallpaper to life'. The torch beam is used to highlight an object in the scene, capturing its shape by pressing the square button on the torch and then as the torch is moved the virtual copy flies around follow. Figure 7 shows a space rocket has been captured from a picture on the wall.

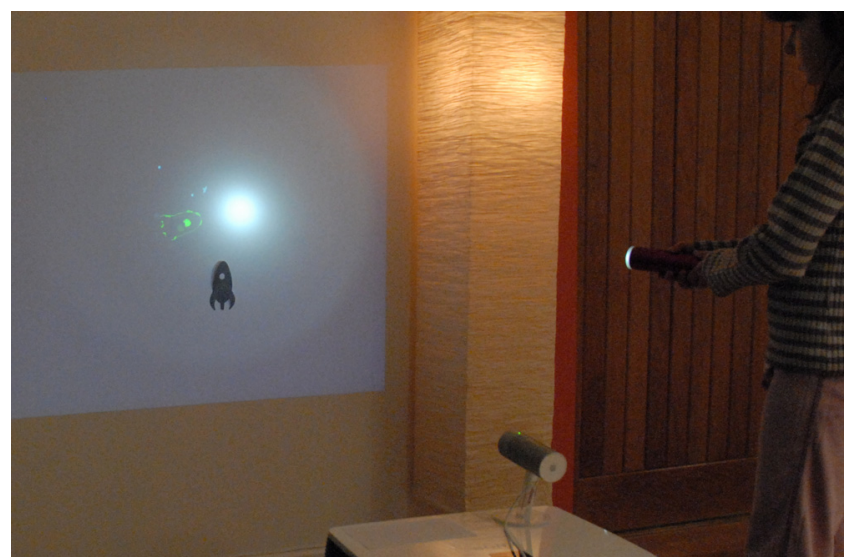

Figure 7 Playing with the Fly sketch

Figure 8 shows each step of the acquisition of the rocket object. It is a very similar process to that used use in Snow. The location of the torch beam is used to create a mask that is applied to the captured background image, extracting and encoding only edges inside it.

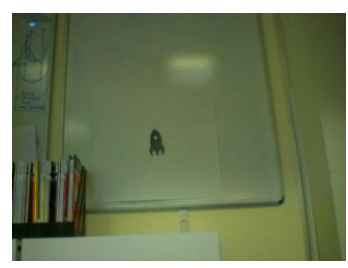

Background

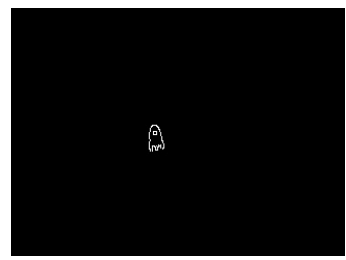

Extracted edges from masked background

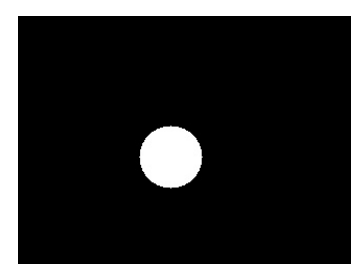

Torch mask

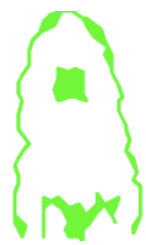

Extracted object (swf)
Figure 8 Extracting background objects for Fly

\subsection{Grid}

Grid was motivated by the tradition of shadow puppets. Here shapes are made with hands or objects in a grid of nine squares allowing complex shapes to be created.

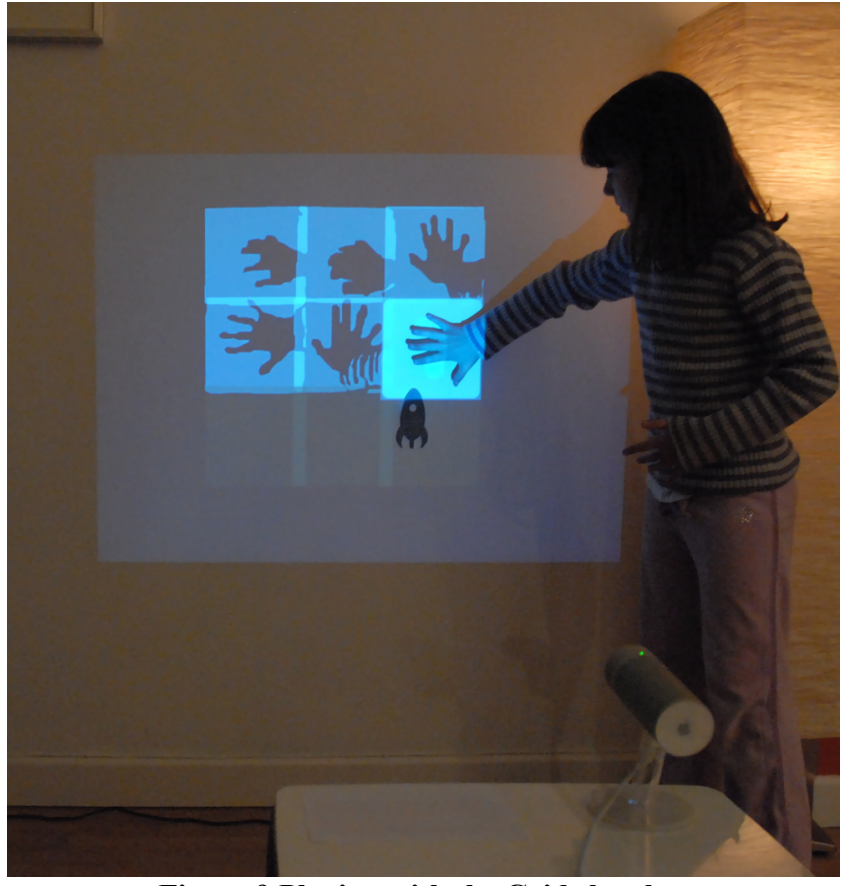

Figure 9 Playing with the Grid sketch

The process is similar to the Snow and Fly applications. Each square is illuminated in turn, counting down from three to zero, with the sound of a camera shutter reinforcing the moment of capture. For each position a new mask is created to isolate the area of interest. Within this area each pixel in the captured image is compared to its equivalent in the background image; where the pixel's hue is sufficiently different it is labelled as part of the foreground object. The edges of the resulting foreground object are extracted and encoded as previously described. For best results the object being captured needs to be relatively well lit against the background.

The original intention had been to look at the shadows cast onto the wall, using the projector as a source of light, rather than the objects themselves. However, as our design has the camera and projector placed together the object would frequently occlude the shadow in the camera's view.

\section{DISCUSSION}

Here we reflect on the success of the Visible Imagination platform and the sketch applications, highlighting challenges and concluding with our vision of how VI can be used in imaginative play. Through our own interactions, demonstrations and an informal observation of an eight-year old child using the system, we can identify a number of issues and opportunities for future examination.

In general we found the use of the torch to be intuitive and fun. The use of visible light meant that where the beam was used to highlight a specific graphic it would become somewhat washed-out. This was an issue in the Scrapbook application, but much less in Fly (where the torch is an attractor) and Game (with the angle-based control). This problem is similar to touch interactions, where the finger or hand obscures the selected item. Schemes to alleviate this include "take-off" proposed by Potter et al [9], where the selection cursor is positioned slightly above the point of contact; this could be adapted for torch interactions.

In the Scrapbook application, where two objects are very close, it becomes difficult to select one or the other. This is a product of the size of the beam relative to the object size. A related 
problem is having certainty that the beam is being tracked; if the torch is too far from the surface its appearance becomes too dim to identify. To alleviate both issues, a pointer could be projected to indicate the tracked beam. It is our preference that the pointer be relatively subtle, for example fairy-dust or crosshairs, such that the beam itself is still seen as the primary means of selection.

In the Menu sketch, no additional pointer is needed as the highlighted object responds to the beam by enlarging and providing audio feedback. With the confirmation required from the button press, false selections were not made.

The capture of shapes from hands and objects is quite successful, although they must be adequately illuminated. The use of a higher resolution camera would further improve the detail of the shapes acquired.

The collocation of the camera and projector, with the user interacting on the same side of the display surface, does present some challenges. Inadvertent obscuring of the projection was seen in our interactions with the system, especially when using with hands in the Grid and Snow sketches. However, the torch interactions do allow the user to stand back, out of the projection volume. In addition this configuration prevents the capture of shadows as we had originally intended; as discussed. While there are drawbacks to integrating the components into a single unit, it does allow a simple installation in the space, giving the flexibility needed for a child's toy.

In future work we would like to exploit obscured projection and allow for real-time interactions with foreground objects [3].

The movement of the torch is interpreted literally in the current system. There is the potential for more abstract gestures to be used, for instance forming a figure-of-eight to initiate an action. These gestures may also be captured in parts of the scene that the camera sees, but are outside the projection area.

The near universal range of the torch, the limited angle of the camera's view and the projection area, make four invisible divisions of the space. Firstly, the region where the torch can be pointed, but neither the camera can view or the projector can display. Secondly, the region within the camera's field of view, but where the projector cannot display; which can used for abstract gestures as discussed. Thirdly, the region outside of the range of the camera, where the projector can display; our configuration attempts to eliminate this case. The fourth region is that where the camera can see and the projector can display; which we clearly seek to maximise. The user's conceptualisation of these regions and their boundaries is crucial for them to understand the system's behaviour. Ghali et al [1] use an audible confirmation of the fourth state. We would like to explore this further, allowing users to conceptualise the space. Molyneaux et al. [6] offer an alternative approach with a steerable projector and camera, which maximises the fourth region.

These six sketches have enabled us to understand the possibilities for interaction with the Visible Imagination system, as currently configured. We have identified a number of specific interaction issues which we will seek to evaluate more formally. Learning from this, we would like to create an engaging toy, to be trialled over a longer period with fully developed applications, beyond the sketches presented here. Our vision for this is a storytelling environment, where children can capture shapes and animations, controlled with the torch, to create stories to be shared across the network with remote family and friends.

\section{ACKNOWLEDGMENTS}

We acknowledge the contribution of Martin Trimby who designed the torch, also of Gary Simpson and Dave Bird who constructed the torch. The Visible Imagination prototype was developed under the Digital Home Venture at BT's Broadband Applications Research Centre.

\section{REFERENCES}

[1] Ghali, A., Boumi, S., Benford, S., Green, J., and Pridmore, T. 2003. Visually tracked flashlights as interaction devices. In Proc. INTERACT. IFIP, September 2003.

[2] Green, J., Schnädelbach, H., Koleva, B., Benford S., Pridmore, T., Medina, K., Harris, E., Smith, H. 2002. Camping in the digital wilderness: tents and flashlights as interfaces to virtual worlds, CHI 2002 Extended Abstracts, 780-781.

[3] Ishii, Y. and Homura, H. 2008. It's fire, you can touch it. Prix Ars Electronica 2008, Linz, Austria.

[4] Kawashima, T., Kida, T. and Niwa, Y. 2006.Takashi's Seasons. ACM Multimedia 2006, 1045-1046

[5] Lee, J., Dietz, P., Aminzade, D., Raskar, R., and Hudson, S. 2004. Automatic Projector Calibration using Embedded Light Sensors. Proceedings of the ACM Symposium on User Interface Software and Technology, October 2004.

[6] Molyneaux, D., Gellersen, H., Kortuem, G. and Schiele, B. 2007. Cooperative Augmentation of Smart Objects with Projector-Camera Systems. In Proc. Ubicomp 2007: $9^{\text {th }}$ International Conference on Ubiquitous Computing, LNCS, Springer-Verlag, September 2007, 501-518.

[7] O'Shea, C. 2007. Out of Bounds. The Design Museum, London. http://www.chrisoshea.org/projects/out-ofbounds/

[8] Olsen, D.R. and Nielsen, S.T. 2001. Laser pointer interaction. CHI 2001, 17-22.

[9] Potter, R.L., Weldon, L.J., and Shneiderman, B. 1988. Improving the accuracy of touch screens: An experimental evaluation of three strategies. Proc. CHI '88, ACM Press, $27-32$.

[10] Ryokai, K., Marti, S. and Ishii, H. 2004. I/O Brush: Drawing with Everyday Objects as Ink. In Proceedings of Conference on Human Factors in Computing Systems (CHI '04).

[11] Summet, J., Flagg, M., Rehg, J.M., Abowd, G.D. and Weston, N. 2006. GVU-PROCAMS: enabling novel projected interfaces. ACM Multimedia 2006: 141-144

[12] Sutton, J.S. and Chatting, D.J. 2008. WISP: Wearable Illumination for Spatialised Play. Night and darkness: Interaction after dark - CHI 2008 Workshop.

[13] Westerdiep, A. de Pong Game. 2006. ArtRock Festival, StBrieuc.

[14] Watson, T. and Lieberman, Z. 2007. Laser Tag 2.0. Graffiti Research Lab. Prix Ars Electronica 2007, Linz, Austria.

[15] Wilson, A. 2005. PlayAnywhere: A Compact Tabletop Computer Vision System, Symposium on User Interface Software and Technology.

[16] Worthington, P. 2005. Shadow Monsters. The Show: Two - Like Nowhere Else, Royal College of Art, London. 